\title{
A Case Report of Toxic Anterior Segment Syndrome, A Rare Complication After Laser in Situ Keratomileusis
}

Aline Ali Ahmad ( $\square$ alineahmad1234567890@gmail.com )

Tishreen University Faculty of Medicine

Ali Othman Hamwi

University of Tartous college of medicine

Rama Basem Hasan

Tishreen University Faculty of Medicine

Sara Othman Hamwi

University of Tartous college of medicine

Hussein Ali Ahmad

University of Tartous college of medicine

Taym Rafik Darwish

Tishreen University Hospital

Original research

Keywords: Case report, TASS, LASIK, inflammatory response.

Posted Date: October 29th, 2021

DOI: https://doi.org/10.21203/rs.3.rs-1016956/v1

License: (9) (i) This work is licensed under a Creative Commons Attribution 4.0 International License.

Read Full License 


\section{Abstract}

Background: Laser in situ keratomileusis (LASIK) is increasingly used worldwide to correct a wide range of refractive errors. Toxic anterior segment syndrome (TASS), a noninfectious inflammatory disease with symptoms and signs that resemble bacterial infection, is a rare complication of LASIK.

Case presentation: We report a case of TASS in a 42-year-old male patient after LASIK surgery in both eyes for hyperopia. Diagnosed clinically.

Discussion and conclusion: Little information about complications after LASIK is available. TASS is a general term used to describe acute, sterile postoperative anterior segment reactions. Patients with TASS will often experience blurry vision with or without pain within 12 to 48 hours after surgery. Steroids have shown to be effective in resolving the inflammatory response, reducing the pain and recovering the vision.

\section{Introduction:}

Laser in situ keratomileusis (LASIK) is increasingly used worldwide to correct a wide range of refractive errors. LASIK consists ofmicrokeratome or femtosecond laser used to create a corneal. Once the flap is created and lifted, laser excimer stromal ablation reshapes the corneal tissue to correct the refractive error and the flap is repositioned at the end of the surgery. [1]

Information about anterior segment inflammatory changes after LASIK is limited. While anterior uveitis has been described as a complication of LASIK with an incidence of $0.18 \%$, [2] the overall incidence of TASS was found to be $0.22 \%$ as reported in a large case series. [3]

Toxic anterior segment syndrome (TASS) is a noninfectious inflammatory disease with symptoms and signs that resemble bacterial infection. TASS can be caused by injury to sensitive structures such as corneal endothelium, iris, and trabecular meshwork. Possible causes may include intraocular solutions, and remnants of materials used to clean and sterilize ophthalmic instruments and other agents. [4] We hereby report a case of TASS after LASIK surgery.

\section{Case Report:}

A 42-year-old man underwent a LASIK surgery in both eyes for hyperopia and it went incompliantly. Systemic and ocular history were unremarkable.

Preoperative refractive error was +7.25 diopter sphere $(\mathrm{D} \mathrm{Sph}) /-0.50$ diopter cylinder $\left(\mathrm{D}\right.$ Cyl) $\times 125^{\circ}$ in the right eye and $+6.50 \mathrm{D} \mathrm{Sph} /-0.50 \mathrm{D} \mathrm{Cyl} \times 63^{\circ}$ in the left eye, and the uncorrected visual acuity was $20 / 200$ in the right eye and 20/100 in the left eye. His corneal topography of both eyes is shown in (shown in figure 1: $a$ and $b$ ).

First day after the surgery has been performed, the patient started on a recipe of the following eye drops: Azithromycin, Genteal lubricant and Fluorometholone. 
On her scheduled postoperative visits Day1, examination of both eyes revealed abrasions on the cornea with bandage contact lenses and a normal anterior segment with an uncorrected visual acuity of 20/50 in the right and 20/63 in the left.

Two days after LASIK, the patient presented with decreased visual acuity in his left eye only (the visual acuity was count fingers on $15 \mathrm{~cm}$ ).

Right eye examination was normal but the left eye showed visualized hypopyon in the anterior chamber with cloudy cornea (shown in figure 2). Posterior segment examination was normal in both eyes.

The patient started on hourly-fortified eye drops (vancomycin and gentamicin) after getting a corneal and anterior chamber culture.

In addition to the eye drops, which failed to improve the patient's condition, the patient started on subconjunctival injections contained of dexamethasone $[8 \mathrm{mg} / \mathrm{ml}]$ and gentamycin $[40 \mathrm{mg} / \mathrm{ml}]$ (to prevent secondary infections).

After two days of steroids, the visual acuity became 20/32 on the right eye and 20/100 on the left eye and after two weeks, it became 20/20 on the right eye and 20/40 on the left eye and the pain and hypopyon resolved (shown in figure 3). Diagnosis of TASS was made.

\section{Discussion And Conclusion:}

There are many complications after LASIK surgery, like under corrections, overcorrections, flap problems, epithelial ingrowth, keratitis, and irregular astigmatism. However, inflammatory reactions after LASIK are not reported enough. [5]

TASS is a postoperative anterior segment sterile reaction. Symptoms include blurry vision after surgery with or without pain.

Clinical findings could include diffuse edematous cornea, endothelial damage, inflammation of anterior segment, hypopyon, dilated/irregular pupil. [6]

There have been different assumptions about uveitis after LASIK and one of them is the abrupt increase and decrease of intraocular pressure after the application of suction ring, which affects like a closed globe injury. Another theory presumed that the shock wave after every excimer application encourages an inflammation reaction and this inflammatory response relates to the depth of ablation, however, there have been authors who do not agree with this theory. [2]

In our case, the patient presented with hypopyon in just one eye, although the bilateral similarity in the refractive error which does not make the depth of ablation a cause of the inflammation.

Preoperative examinations including slit lamp examination and fundoscopy were normal. Ocular pressure exam with air-puff tonometer was in the normal range before and after the LASIK, which exclude the 
ocular pressure hypothesis.

In the refractory clinic, this complication has been observed for the first time.

The corneal and anterior chamber culture which were taken before the start of the fortified eye drops were negative, which exclude the bacterial cause, although we can consider the viral infection, however with the resolution of the hypopyon with steroids, the viral cause could be excluded, and the inflammatory reaction could be considered.

TASS can be caused by many materials such as remnants left behind after sterilization of surgical instruments, irrigating solutions, osmotic or ionic composition. Also it can be due to intraocular drugs with preservatives, stabilizing agents, endotoxins, heavy metals at overdoses. The ointments and eye drops after surgery are involved too. [7]

In most cases, the cause of TASS remains unknown even after a thorough investigation, and knowing the causative agent can sometimes be hard.

The patient's clinical symptoms and subsequent response to topical steroids medications were consistent with TASS, we hereby emphasis on considering TASS as a complication after LASIK in any patient developing an inflammatory response and to treat it as soon as possible with steroids due to its rapid response and recovery as reported by our patient.

\section{Abbreviations}

LASIK: Laser in situ keratomileusis

TASS: Toxic anterior segment syndrome

D: diopter

Cyl: cylinder

Sph: sphere

\section{Declarations}

Ethics approval and consent to participate:

Not applicable.

\section{Consent for publication:}

Written informed consent was obtained from the patient for publication of this case report and any accompanying images. A copy of the written consent is available for review by the Editor of this journal. 
Availability of data and materials:

The authors declare that the data supporting the findings of this study are available within the article and its supplementary information.

\section{Competing interests:}

The authors declare that they have no competing interests.

\section{Funding:}

Not applicable.

\section{Authors' contributions:}

$\mathrm{AH}$ made the conception and design of the work, analyzed and interpreted the patient data, was a major contributor in writing the manuscript. AA made the conception, analyzed and collected the data and was a major contributor in writing the manuscript and is the corresponding author. $\mathrm{RH}$ analyzed the data and was a contributor in writing the manuscript. SH analyzed the data and was a contributor in writing the manuscript. HA analyzed the data and was a contributor in writing the manuscript. TD substantively revised the work.

\section{Acknowledgements:}

Not applicable.

\section{Authors' information:}

AA

00963933545731

Ophthalmology resident at Tishreen university hospital

$\mathrm{AH}$

00963951736713

University of Tartous College of medicine

$\mathrm{RH}$

00963932821255

Ophthalmology resident at Tishreen university hospital

$\mathrm{SH}$ 
00963988072424

University of Tartous College of medicine

HA

00963956319953

University of Tartous College of medicine

TD

00963933880040

Head chef of ophthalmology department at Tishreen university hospital

\section{References}

[1] Khoueir, Z., N. M. Haddad, A. Saad, E. Chelala, and E. Warrak. "Traumatic flap dislocation 10 years after LASIK. Case report and literature review." Journal francais d'ophtalmologie 36, no. 1 (2013): 82-86.

[2] Parmar, Pragya, Amjad Salman, M. Rajmohan, and Nelson CA Jesudasan. "Fibrinous anterior uveitis following laser in situ keratomileusis." Indian journal of ophthalmology 57, no. 4 (2009): 320.

[3] Sengupta, Sabyasachi, David F. Chang, Rajiv Gandhi, Hemal Kenia, and Rengaraj Venkatesh. "Incidence and long-term outcomes of toxic anterior segment syndrome at Aravind Eye Hospital." Journal of Cataract \& Refractive Surgery 37, no. 9 (2011): 1673-1678.

[4] Park, Choul Yong, Jimmy K. Lee, and Roy S. Chuck. "Toxic anterior segment syndrome-an updated review." BMC ophthalmology 18, no. 1 (2018): 1-9.

[5] Perez-Santonja, J. J., Sakla, H. F., Cardona, C., Ruiz-Moreno, J. M., \& Alió, J. L. (1998). Subclinical inflammation after laser in situ keratomileusis. Journal of Cataract \& Refractive Surgery, 24(8), 10591063

[6] Park, Choul Yong, Jimmy K. Lee, and Roy S. Chuck. "Toxic anterior segment syndrome-an updated review." BMC ophthalmology 18, no. 1 (2018): 1-9.

[7] Cetinkaya, Servet, Zeynep Dadaci, Hüsamettin Aksoy, Nursen Oncel Acir, Halil Ibrahim Yener, and Ekrem Kadioglu. "Toxic anterior-segment syndrome (TASS)." Clinical Ophthalmology (Auckland, NZ) 8 (2014): 2065.

\section{Figures}



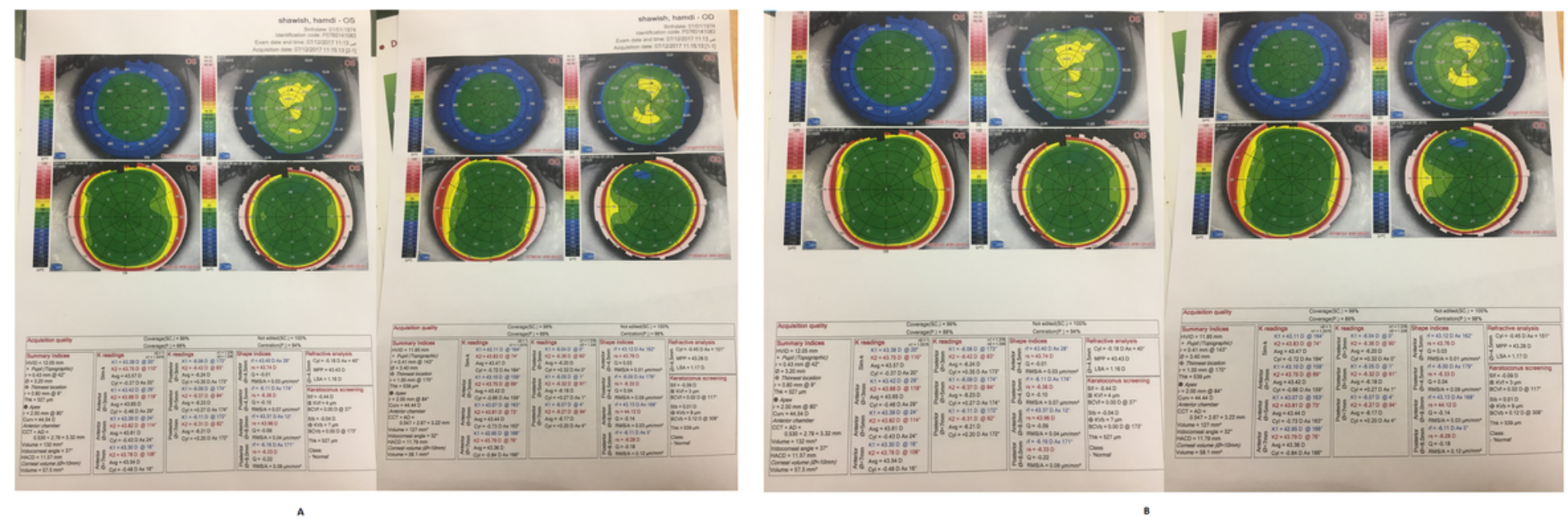

\section{Figure 1}

a, corneal topography of the right eye shows normal shape and measurements of the cornea (the thickness was $539 \mu \mathrm{m}$ and the curve was 44.44D, there wasn't any evidence of corneal ectacia and the Sim-K readings shows astigmatism $-0.72 \mathrm{D}$ at the axis $164^{\circ}$ and average of keratometer $43.47 \mathrm{D}$,there wasn't any abnormalities or scars on the cornea). b, corneal topography of the left eye shows normal shape and measurements of the cornea ( the thickness was $527 \mu \mathrm{m}$ with 44.54D, there wasn't any evidence of corneal ectacia and the Sim-K readings shows astigmatism - $0.37 \mathrm{D}$ at the axis $20^{\circ}$ and average of keratometer 43.57D, there wasn't any abnormalities or scars on the cornea). 


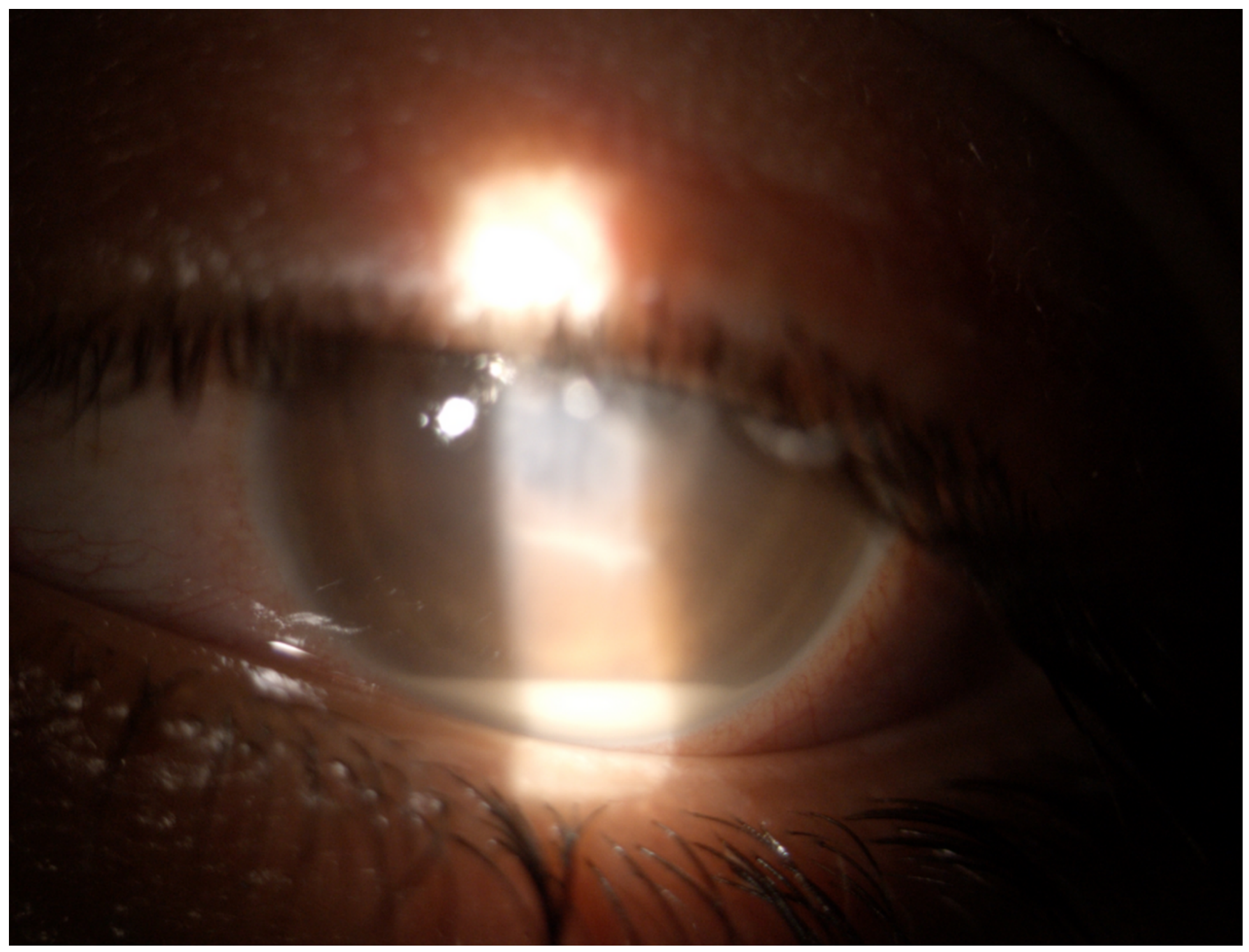

Figure 2

a slit lamp examination of the left eye after two days shows the hypopyon in the anterior chamber and the cloudy cornea in the left eye. 


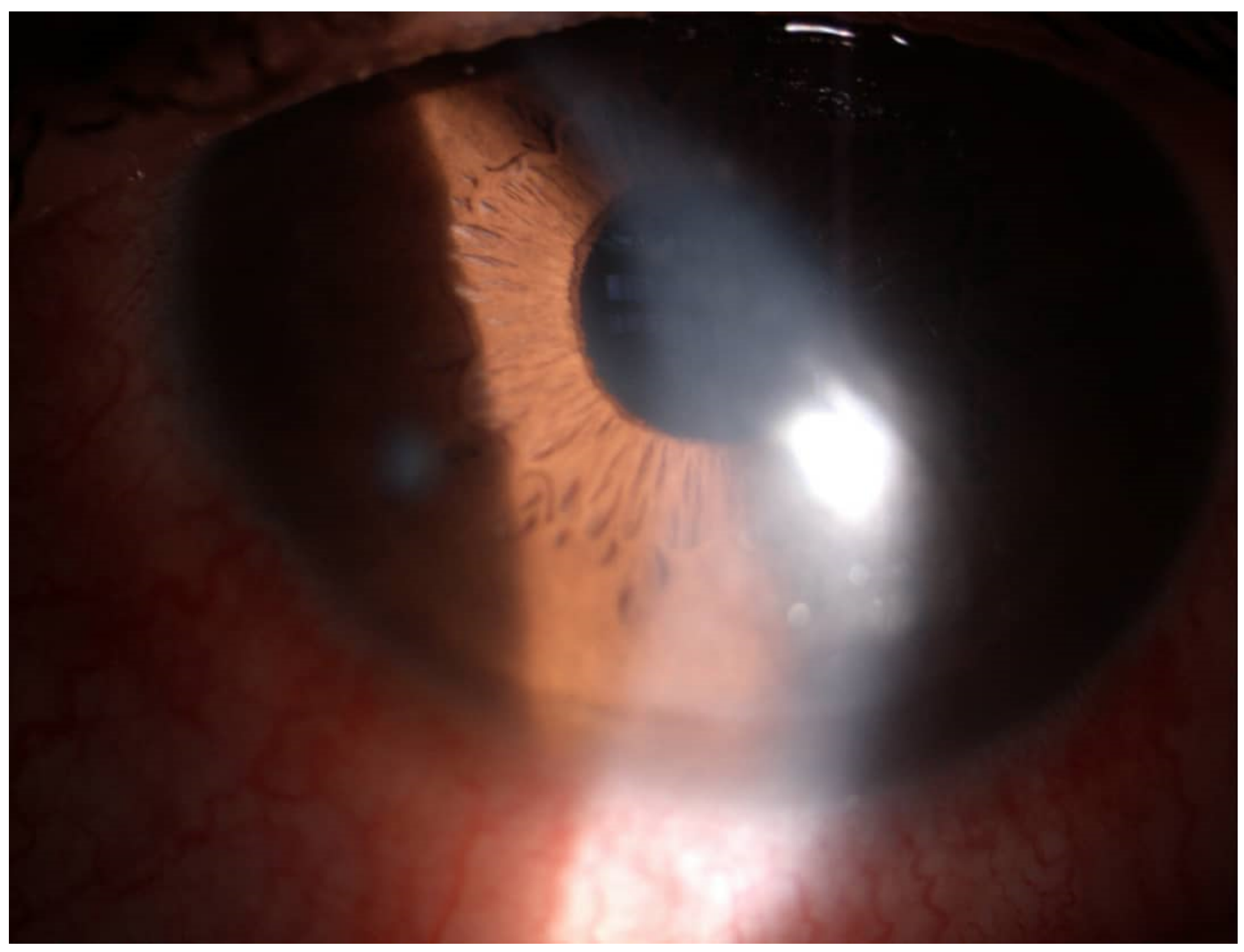

Figure 3

a slit lamp examination of the left eye shows the anterior chamber had become clear, the hypopyon got resolved and the cloudiness of the cornea had reduced a lot.

\section{Supplementary Files}

This is a list of supplementary files associated with this preprint. Click to download.

- carechecklist2.pdf 